\title{
Perceptible MOS Factor Achieved During Voice Transmission in IEEE 802.1Q Standard for Video Conferencing
}

\author{
P. Thangaraj, PhD. \\ Professor \& Head \\ Bannari Amman Inst of Tech \\ Erode
}

\author{
K. Sakthi Sudhan, \\ A. L. Karthika \\ Research Scholars \\ Bannari Amman Inst of Tech \\ Erode
}

\author{
S. N. Sivanandam, PhD. \\ Professor (Rtd) \\ Karpagam College of Engg \\ Coimbatore
}

\begin{abstract}
In the upcoming $4 \mathrm{G}$ networks can provide better quality parameters values than $3 \mathrm{G}$ networks. Video conferencing is IP based connectivity to $\mathrm{N}$ number of users in the geographical coverage area in order to obtain good quality parameters like throughput, delay, jitter, traffic pattern in sending/receiving end of users. Streaming protocol for audio/video conferencing over VoIP based protocols over VLAN scenario.
\end{abstract}

Keywords_-VLAN, MOS, H.323, Streaming

\section{INTRODUCTION}

4G describes the fourth generation of wireless services which is extended from $3 \mathrm{G}$ technology. It offers more Bandwidth and services than $3 \mathrm{G}$. Over internet protocol $4 \mathrm{G}$ offers high quality audio and video streaming. The $4 \mathrm{G}$ wireless service can offer downloading speed ten times than the $3 \mathrm{G}$ service. VoIP service can perform well in 4G technology.

In traditional LAN, the nodes are connected using switches or hubs or repeaters. The device propagates the data received by it throughout the network. Communication with devices in another LAN segment requires routers. If two nodes are transmitting at same time collision will occur and the transmitted data will be lost. The information about collision will be propagated throughout the network through hubs and repeaters. Then the data want to be retransmitted again from the corresponding node after the collision is resolved. This causes the wastage of time and resources. The limitations of the LAN are i) As the number of nodes connected to the network increases the data transfer rate to each node will decrease. ii) Fault in the network causes the nodes to lose data and makes access to various nodes impossible. iii) Security offered is less. iv) Resource consumption is more. v) Latency and delay for the transmission of data is high. Vi) Large network maintenance is difficult. Vii) Cost required is high. The limitation of the LAN can be rectified somewhat by VLAN.

\section{VLAN}

The main goal of VLAN is to break up the broadcast domain without using routers. Virtual Local Area Network (VLAN) the network manager will logically segment the LAN into different broadcast domain. The physical segmentation is not done. Since it is a logical segmentation there is no need that the node wants to be placed together. The nodes on different floor of the same building or at different building can come under same VLAN. VLAN can be configured through hardware or software. This increases the flexibility of VLAN.
In this the nodes can move to another location and also it can stay in the same VLAN without any hardware reconfiguration. VLAN can group devices logically into single broadcast domain. This allows to confine the broadcast traffic only to that specific Workgroup. VLAN segmentation can be done based on switch port or Media Access Control (MAC) address or IP subnets. The communication between the switches makes large VLAN. On the basis on the segmentation of VLAN implicitly or explicitly communication is possible. The benefits of VLAN are a) Increased security b) Improved performance. c) Simplicity of software configuration and network tuning. d) Improved manageability. e) Independent of physical topology.

\section{VoIP Transmission Technology}

Voice over IP is a fast growing technology in the world of communication. VoIP serves IP network based voice and video communication. VoIP allows the users to make voice communication through broadband internet connection instead of a traditional telephony system. With the advantages of VoIP services including low cost and extra feature results in its popularity. Traditional PSTN system is a circuit switched network which requires dedicated line for communication. VoIP uses the internet for the transmission of packet via packet switched network. A VoIP system has three components namely CODEC, Packetizer, Payout Buffer. At the transmitter voice signals are compressed then encoded and converted into digital packets which are transmitted through the internet. At the receiver digital packets are converted back to analog signals. The benefits of VoIP service are mentioned below. I) VOIP access eliminates the difficulty associated with fixed line payment. II) VOIP allows service anywhere through Internet III) IP telephone is easy to upgrade the infrastructure for the future. IV) It supports all forms of communication standardization. V) Access terminal portability and its consequences such as it are being personal and cost effective. VI) The new business model used to commercialize it. VII) New and enhanced service provisions.

\section{QoS Parameters in video call setup}

Voice quality is a major issue in VoIP. When the voice comes together with IP quality problem may arise because VoIP is asynchronous and real time application. Voice quality is a function of packet delay variation, end to end delay, jitter and MOS.

a) Packet Delay Variation: Packets are transmitted as a stream. Packet delay variation is the one way delay between the selected packets in flow with any lost packet being 
ignored. Inter Packet delay variation also specifies the change in inter-packet spacing between transmission and reception.

\section{b) End to End delay}

End to End is the time taken for a packet to be transmitted from the source to destination. The end to end delay includes transmission delay, propagation delay, and processing delay. In real time data end to end delay is to be guaranteed.

c) Jitter

Jitter is the variation of signal being received. In VoIP jitter is the variations in the time between the packets are arriving. The causes are network conjunction, time drift, route change.

\section{d) MOS}

In voice communication Mean Opinion Score (MOS) is a numerical value that gives the quality of the received voice signal. It indicates whether the received signal is good or bad. The MOS value that obtained in this work indicates fair voice communication.

\section{RELATED WORKS}

There are many papers are there based on VoIP service in WLAN. The authors are mainly concerned in the performance of VoIP in WLAN environment. [1] K. Sakthisudhan et al analysed the performance of the video streaming service in in indoor and outdoor WIFI. [2] M. Atif Qureshi et al investigated the performance in wimax and wifi. [3] Ye Chen et al proposed a novel power management technique for stations and services that use contention-based channel access which is Unscheduled Power Save Delivery (UPSD) and investigate the performance of UPSD in combination with EDCA mechanism. [4] Sajal K. Da et al analysed the performance of the H.323 call set-up procedure over wireless links using a simple call model under two modes of operations, namely, Regular and Fast Connect. [5] Wei Wang et al proposed and investigate M-M scheme that can improve the VoIP capacity by close to $100 \%$ without changing the standard 802.11 CSMA/CA protocol. [6] Deyun Gao et al introduced the use of the service differentiation provided by the new IEEE 802.11e standard to solve the bottleneck problem and improve voice capacity. [7] An Chan et al investigated several solutions to improve the VoIP capacity. [8] JengFarn Lee et al studied the behaviour of voice over IP traffic in IEEE 802.11 wireless networks. [9] Lin Cai et al did the survey of recent advances in voice over WLAN voice capacity analysis, call admission schemes, and medium access control (MAC) layer quality of service (QoS) enhancement mechanisms. [10] Rizik Al-Sayyed et al analysed the maximum number of simultaneous Voice over Internet Protocol (VoIP) that would support under the G.711 and G.729 CODEC standards and examined the VoIP user capacity using the G.729 CODEC standard along with background traffic.

\section{RESULTS AND DISCUSSION}

In this VLAN scenario area is of $100 \times 100$ metres. Fig 1 shows the Vlan scenario. Node $0-5$ are the client nodes. Node 0-2 are connected to the Ethernet switch 1 and node 3- are connected to switch. Switches are connected to an Ethernet router. The server provides VoIP service which is shared to all the clients through a router.

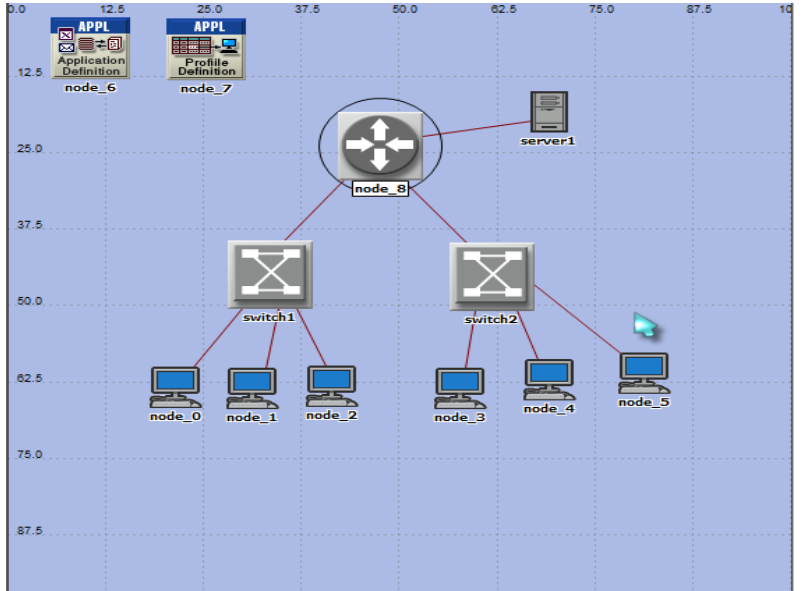

Fig1 OPNET snapshot of wired VLAN

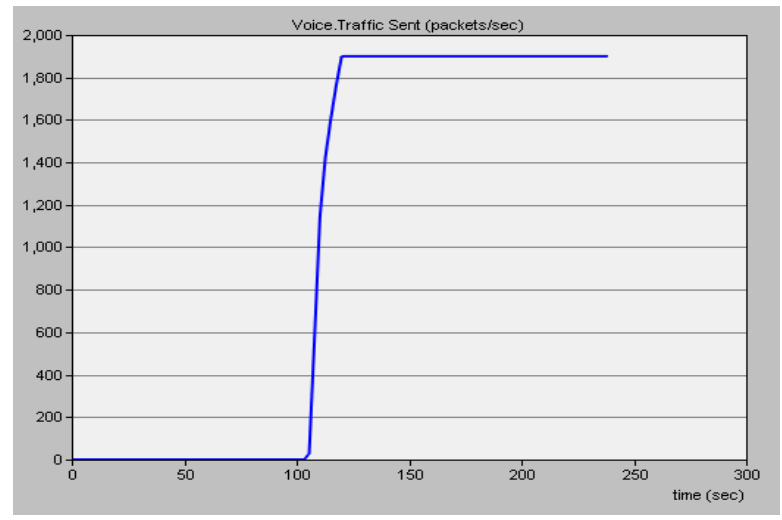

Fig 2 VOIP traffic sent in the VLAN

Fig 2 shows the amount of VoIP data sent on the VLAN network. Network traffic is the number of packets sent and received in a network. Traffic sent includes the number of packets sent from the server to destination.

I. TABLE

\begin{tabular}{|l|l|}
\hline \multicolumn{1}{|c|}{ Parameters } & \multicolumn{1}{|c|}{ VLAN } \\
\hline Physical layer parameters & $\begin{array}{l}\text { IEEE 802.1Q with Ethernet } \\
\text { network }\end{array}$ \\
\hline Data rate & 100 Mbps \\
\hline Signalling protocol & $\begin{array}{l}\text { H.323 direct end point call } \\
\text { signalling mode }\end{array}$ \\
\hline Router configuration & $\begin{array}{l}\text { Atm4_ethernet2_gtwy fixed } \\
\text { point IP router }\end{array}$ \\
\hline Switch & $\begin{array}{l}\text { Ethernet } \\
\text { Tr16_switch }\end{array}$ \\
\hline VLAN server & $\begin{array}{l}\text { Ethernet IP severs } \\
\text { Operational mode is a MAC } \\
\text { layer (full duplex) }\end{array}$ \\
\hline Working stations & $\begin{array}{l}\text { Ethernet workstation_adv } \\
\text { client to server model }\end{array}$ \\
\hline
\end{tabular}


With VLAN server capability of inter connectivity through Ethernet connection at 10,100, 1000 Mbps. And it's supported for TCP/IP , UDP/IP based wired IP protocols. The speed of operation link by its data rate. The ethernet gateway router has provided IP based connectivity of two user interfaces at 10 Mbps. It provides a fixed amount of the TTL (time to Time Live) to route the packets via forwarding RIP (routing Information protocols).

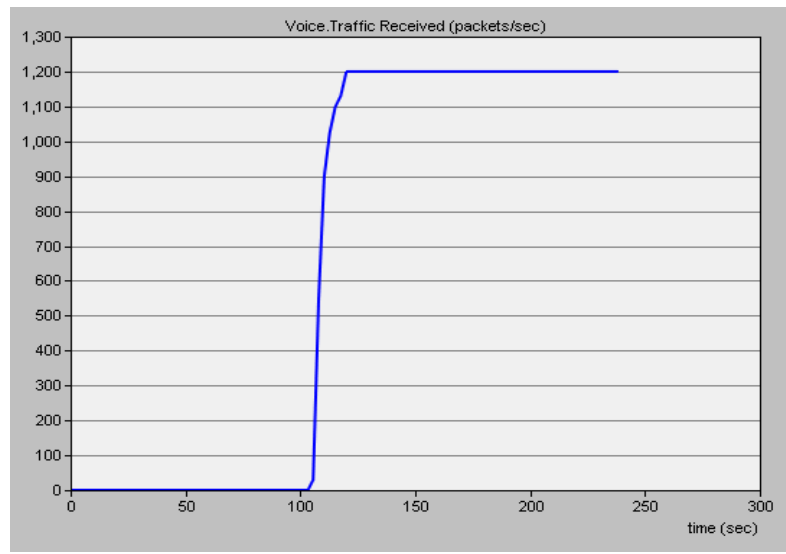

Fig 3 VoIP traffic received in Vlan

The below Fig 3 shows the traffic received by the VLAN clients. The queuing of packets can be done by the routers. Packet delay variation is the difference in one way delay of the packets. Packet delay variation depends on the network load/traffic.

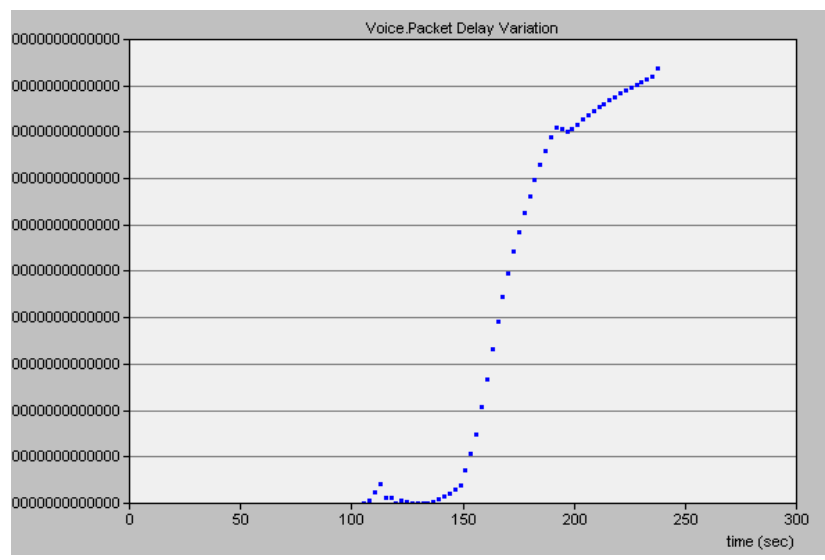

Fig 4 VoIP packet delay variation

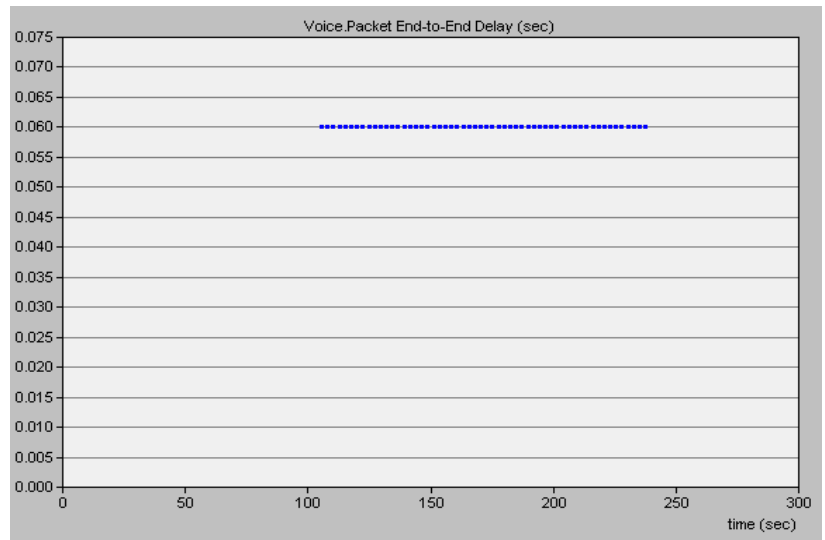

Fig 5 VoIP packet end to end delay (Sec)
Fig 4,5 and 6 shows the delay and jitter parameter values. The end to end delay is the time taken for a packet to be transmitted from the source to destination. End to end delay depends on processing delays, propagation delay and transmission delay. The result value of packet delay, jitter are almost zero and end to end delay is obtained constant value is 0.60 in during voice transmission period.

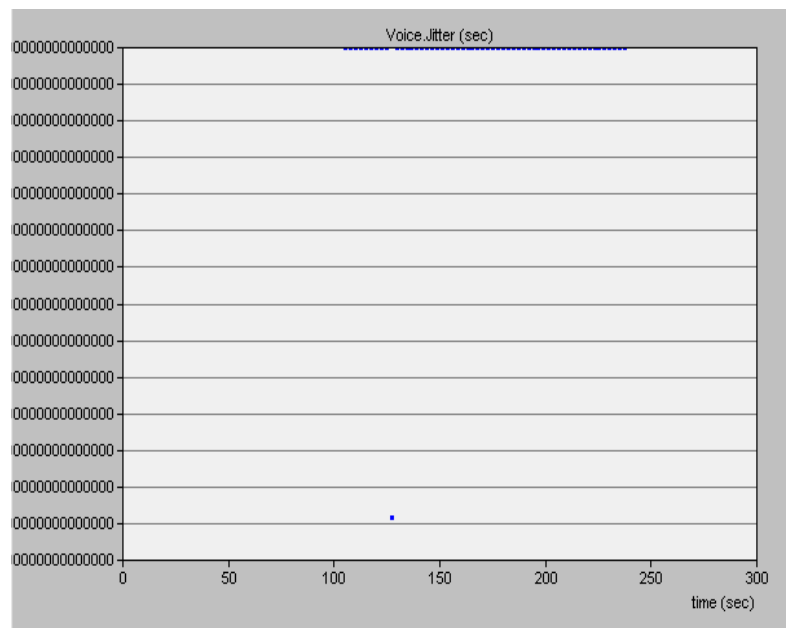

Fig 6 VoIP transmission of jitter

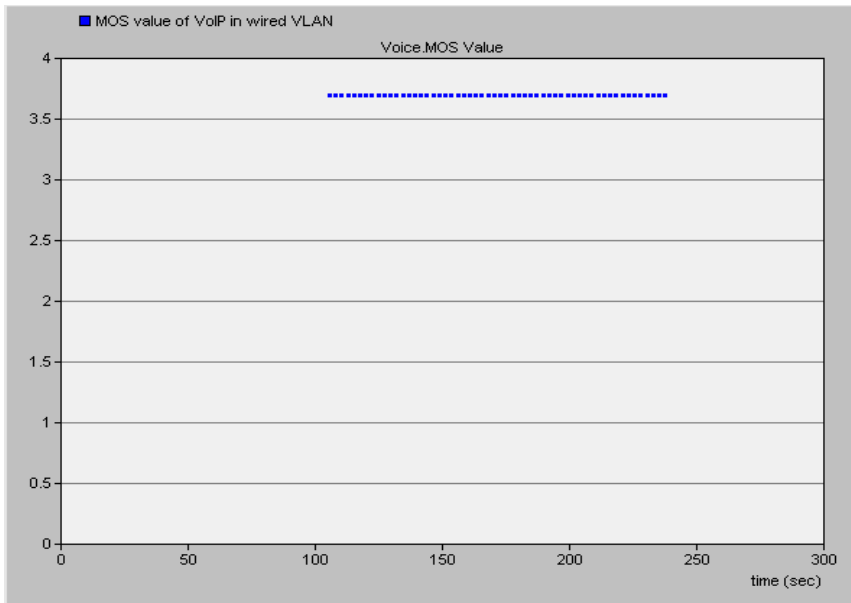

Fig 7 MOS factor for Voice transmission

From Fig 7 represents MOS factor in the voice communication. It indicates whether the received voice signal is good or bad. The MOS value between 3.5 and 4 gives 1 is quality of signal is good and also provide impairment is perceptible and not annoying results.

\section{CONCLUSION}

In this paper, OPNET scenario for campus coverage network area is 100 by 100 located the ethernet working model attached with the router, switches are real time configuration. In this scenario describes the MOS factor in order to obtain the values between 3.5 and 4 . The impairment of the scenario result is practically perceptible and not annoying. And also analysed the quality factors are perfectly good for voice transmission in video conferencing applications. The analytical results were validated by our experimental measurements. 


\section{REFERENCES}

[1]K.SakthiSudhan, P.Thangaraj, A.L.Karthika, “Achieving Quality of Metric for video streaming service in warehouse application with coexisting of IEEE $80.11 \mathrm{a} / \mathrm{b} / \mathrm{g}$ standards special issue of IJCA on advance computing and communication technologies for HPC applications PP 27-31 June 2012.

[2] M. Atif Qureshi, Arjumand Younus, Muhammad Saeed, Farhan Ahmed Sidiqui, Nasir Touheed, M. Shahid Qureshi "Comparative Study of VoIP over WiMax and WiFi" IJCSI International Journal of Computer Science Issues, Vol. 8, Issue 3 PP 433-437 May 2011.

[3] Ye Chen, Natt Smavatkul, Steve Emeott "Power Management for VoIP over IEEE 802.11 WLAN"

[4] Sajal K. Das, Kalyan Basu "Performance Optimization of VoIP Calls over Wireless Links Using H.323 Protocol" IEEE TRANSACTIONS ON COMPUTERS, VOL. 52 PP 742-752 June 2003.

[5] Wei Wang, Soung Chang Liew, Victor O. K. Li, "Solutions to Performance Problems in VoIP Over a 802.11 Wireless LAN" IEEE TRANSACTIONS ON VEHICULAR TECHNOLOGY, VOL. 54 PP 366-384 January 2005.
[6] Deyun Gao, Jianfei Cai, Chuan Heng Foh, Chiew-Tong Lau, King Ngi Ngan "Improving WLAN VoIP Capacity Through Service Differentiation" IEEE TRANSACTIONS ON VEHICULAR TECHNOLOGY, VOL. 57 PP 465-474 January 2008.

[7] An Chan, Soung Chang Liew "Performance of VoIP over Multiple Co-Located IEEE 802.11 Wireless LANs" IEEE TRANSACTIONS ON MOBILE COMPUTING, VOL. 8 PP 1063-1076 August 2009.

[8] JengFarn Lee, Wanjiun Liao, Jie-Ming Chen, and HsiuHui Lee "A Practical QoS Solution to Voice over IP in IEEE 802.11 WLANs" IEEE Communications Magazine PP 111-117 April 2009.

[9] Lin Cai , Yang Xiao , Xuemin (Sherman) Shen , Lin Cai, Jon W. Mark "VoIP over WLAN: Voice capacity, admission control,QoS, and MAC" INTERNATIONAL JOURNAL OF COMMUNICATION SYSTEMS PP 491-508 January 2006.

[10] Rizik Al-Sayyed, Colin Pattinson, and Tony Dacre "VoIP and Database Traffic Co-existence over IEEE 802.11b WLAN with Redundancy" World Academy for Science, Engineering and Technology PP 289-294, 2007. 